\title{
UNA ESTRATEGIA PARA OBTENER REGIONALIZACIONES BIÓTICAS FIABLES A PARTIR DE DATOS INCOMPLETOS: EL CASO DE LOS ESCARABEIDOS (COLEOPTERA, SCARABAEINAE) IBÉRICO-BALEARES
}

\author{
J. Hortal ${ }^{1 *}$, J. M. Lobo ${ }^{1}$ y F. Martín-Piera ${ }^{1}$
}

\begin{abstract}
RESUMEN
Se presenta un método para obtener regionalizaciones basadas en los organismos a partir de datos corológicos incompletos, utilizando como ejemplo los escarabeidos ibéricos (Col., Scarabaeinae). A partir de las unidades territoriales bien muestreadas, realizamos en primer lugar un análisis de ordenación, en el que se identificaron cuatro ejes faunísticos. Posteriormente, los valores de estos ejes se extrapolaron a todo el territorio ibérico mediante Modelos Lineales Generalizados. Con los valores predichos para estos ejes se desarrolló una matriz de distancias faunísticas. A partir de ella, y de las distancias geográficas entre unidades territoriales, se obtuvo una nueva matriz de distancias faunístico-geográficas. Esta matriz fue utilizada para realizar la clasificación final de todo el territorio, obteniéndose ocho regiones faunísticas diferentes. Finalmente, se comentan los resultados obtenidos, identificando las diferencias en su composición y riqueza regional y local.

Palabras clave: regionalización, clasificación, ordenación, modelos predictivos, Scarabaeinae, Península Ibérica.
\end{abstract}

\begin{abstract}
A strategy to obtain reliable biotic regionalizations from incomplete data: the case of IberoBalearic dung beetles (Coleoptera, Scarabaeinae)

A method to obtain regionalizations based in the organisms from incomplete distributional data is presented, using Iberian scarabaeine (Col., Scarabaeinae) as an example. A first ordination analysis was carried out using the well-sampled territorial units, where four faunistic axes were identified. Then, the scores of these axes were extrapolated to the whole Iberian territory by means of General Linear Modelling. A faunistic distance matrix was developed using the predicted values for the four axes. Using this matrix and the geographic distances among territorial units, a new matrix, reflecting both the compositional and geographic distances was obtained. This matrix was used to carry out the final classification of all the territory, where eight different faunistic regions were obtained. Finally, the results are briefly discussed, and their composition and local and regional richness are described.
\end{abstract}

Key words: regionalization, classification, ordination, predictive models, Scarabaeinae, Iberian Peninsula.

Departamento de Biodiversidad y Biología Evolutiva. Museo Nacional de Ciencias Naturales. C/ José Gutiérrez Abascal, 2. 28006. Madrid, España. *e-mail menjh521@mncn.csic.es 


\section{Introducción}

La regionalización es el proceso de agrupar $u$ ordenar la información acerca de un número determinado de unidades territoriales, con el propósito de representar las similitudes y relaciones entre ellas (Bailey et al., 1978; Bailey, 1996). Como consecuencia de esta acción pueden obtenerse representaciones cartográficas útiles para discriminar los diferentes tipos de regiones ambientales o los conjuntos de organismos que comparten su distribución espacial, además de permitir describir su extensión y ubicación. Tanto la determinación de los puntos con mayor riqueza de especies (áreas de superposición cartográfica; Rapoport, 1975), como la caracterización de las regiones de un territorio a partir de sus diferencias ambientales o de composición faunística, han sido cuestiones abordadas tradicionalmente por la Biogeografía (Hengeveld, 1990) con el fin de representar los principales patrones de distribución de los organismos y estudiar los procesos ecológicos y/o históricos que han propiciado su actual distribución. El interés por los asuntos relacionados con la conservación ha aportado un nuevo punto de vista sobre estos asuntos, de modo que reconocer los principales patrones en la distribución territorial de un grupo de organismos o de las características ambientales, es ahora relevante para diseñar nuestras redes de reservas, de manera que se maximice su representatividad (la proporción de la biodiversidad total representada por la red de reservas; ver Austin \& Margules, 1986).

Existen clasificaciones territoriales que utilizan diversa información ambiental para reconocer las distintas ecoregiones o ecosistemas (Bailey, 1996; ejemplos de esta aproximación en el territorio español pueden verse en de Pablo \& Pineda, 1985; de Pablo et al., 1987 y 1988; Elena Rosselló et al., 1990, 1993 y 1997). La utilización en conservación de esta clasificación orientada hacia los sistemas ecológicos (Faith et al., 2001), se basa en discriminar unidades ambientales sin considerar la información sobre los actores (las poblaciones y las especies) que habitan en ellas, una aproximación que no parece garantizar la protección de estos últimos (Araújo et al. 2001; Mac Nally et al., 2002). Si unidades ambientalmente similares pueden contener conjuntos faunísticos diferentes, debido a la actuación de factores distintos de los puramente ambientales, tampoco parece posible establecer una correspondencia estricta entre un tipo de unidad ambiental y los procesos que en ella tienen lugar. Tras muchos años de descripción de procesos ecológicos locales, estos han resultado ser demasiado contingentes entre lugares ecológicamente similares como para predecir qué procesos serán importantes en qué tipos de sistemas a partir de nuestros conocimientos actuales (Lawton, 1999). Ante este panorama, y si la estructura de los sistemas ecológicos es consecuencia de la actuación de las especies que los integran, parece recomendable que el esfuerzo en investigación y conservación de la biodiversidad no se centre únicamente en los ecosistemas, sino también en las especies y ensambles (conjuntos de especies) que los habitan.

Desde el punto de vista de la conservación, la subdivisión de un territorio en unidades faunística o florísticamente homogéneas puede ser útil a la hora de predecir la distribución de las especies, proponer una red de espacios protegidos capaz de proteger la diversidad biológica, o diseñar una estrategia de restauración o reintroducción. Para estimar la distribución de las especies se asume que la especie encontrada en una región determinada, generalmente definida por criterios florísticos o fitosociológicos, se encuentra presente en toda el área ocupada por ella (Scott et al., 1993). La selección de áreas de conservación para un grupo taxonómico determinado mediante las regionalizaciones, supone que una elección de enclaves que refleje la heterogeneidad espacial en la composición de sus comunidades sería capaz de representar adecuadamente su diversidad biológica (Austin, 1999; Ferrier, 2002; Ferrier et al., 2002). Se trata, así, de realizar clasificaciones territoriales específicas para cada grupo biológico que, siendo capaces de describir la variación espacial en su composición, permitan, con el concurso de la información sobre la distribución de otros atributos de la diversidad biológica como la riqueza de especies, la rareza o la endemicidad, delimitar la ubicación de los enclaves con mayor valor de conservación.

En el caso concreto de la vegetación, las regionalizaciones florísticas se han usado directamente para delimitar redes de espacios de conservación globales, debido a su supuesta capacidad para integrar muchos de los atributos físicos y biológicos de un área. (Scott et al. 1993; Powell et al., 2000). Esta ha sido, en esencia, la aproximación seguida mediante las clasificaciones territoriales construidas a partir de los datos sobre cobertura del suelo o vegetación potencial (Izco, 1984; Rivas-Martínez, 1987; Gavilán et al., 1998), las cuales han servido de base para el diseño de nuestra actual red de reservas (ver, por ejemplo, http://www.faunaeur.org/PUBLIC/ComBioDivStrat. pdf o http://www.mma.es/conserv_nat/planes/basensio/index.htm). 
Otra de las aplicaciones potenciales de las regionalizaciones basadas en los organismos es la planificación de muestreos y la selección de las localidades de colecta (Bunce et al., 1983, 1996; Balmford \& Gaston, 1999; Fisher, 1999), una cuestión metodológicamente muy similar a la de seleccionar la localización de los espacios a proteger. Se trata pues de elegir el emplazamiento de las localidades de muestreo al objeto de maximizar la diversidad de un grupo de organismos que podemos colectar en un territorio determinado, de modo que si las localidades de colecta son ubicadas en cada una de las clases territoriales aumentaría la probabilidad de obtener una representación fiable de todos los conjuntos faunísticos existentes.

Existen diversos intentos de regionalización basados en los organismos que habitan la Península Ibérica. Sin embargo, es difícil realizar comparaciones efectivas entre ellas, ya que han utilizando unidades territoriales y escalas muy variables (cuencas, regiones administrativas, regiones naturales, cuadrículas UTM, etc.). Dichas regionalizaciones han utilizado la información biológica disponible sobre plantas y vertebrados (Sainz Ollero \& Hernández Bermejo, 1985; Doadrio, 1988; Real et al. 1992 y 1996; Palomo et al., 1994; Moreno Saiz et al., 1998; Vargas et al., 1998; Márquez et al., 2001; García-Barros et al., 2002), pero una característica común de estos estudios es la ausencia de análisis que permitan conocer si los inventarios de cada unidad territorial son el resultado de un esfuerzo de colecta lo suficientemente intenso y son, por lo tanto, fiables. Debido a ello, la información corológica disponible sobre los invertebrados es rara vez utilizada para este propósito (ver, sin embargo, Viejo et al., 1992; Hermida et al., 1994; Rodríguez et al., 1997; Puente et al., 1998). Estimar la variación faunística de un grupo de organismos en un territorio requiere utilizar la información disponible sobre la distribución de las especies. Aunque en ocasiones las regionalizaciones se han basado en el criterio de expertos (ver por ejemplo Morrone, 2001), lo más pertinente es utilizar los datos faunísticos de distintas unidades territoriales, una vez estimada su fiabilidad, para realizar clasificaciones utilizando distintas técnicas estadísticas de análisis multivariante. Sin embargo, obtener información biológica fiable suele ser posible, tan sólo, cuando la escala y la extensión geográfica son grandes, pues a escalas pequeñas (ver por ejemplo Wohlgemuth, 1996 y las referencias en su interior), es muy difícil extraer regiones geográficas aceptables debido a que la información sobre la distribución de los organismos es escasa y espacial- mente sesgada (Haila \& Margules, 1996; Dennis \& Hardy, 1999; Lobo \& Martín-Piera, 2002).

Una alternativa razonable a esta falta de información biológica consiste en extrapolar o predecir la distribución de la variación faunística (Lobo \& Hortal, 2003). Las distintas técnicas predictivas existentes (Guisan \& Zimmermann, 2000) pueden utilizarse para modelizar individualmente cada una de las especies de un grupo de organismos al objeto de construir con estos datos extrapolados una regionalización fiable (Peters \& Thackway, 1998). Sin embargo, los problemas derivados de las predicciones de distribución individuales son múltiples (Ferrier, 2002; Hortal \& Lobo, 2002). Por una parte, el efecto de sus errores se multiplica cuando se trabaja con los valores calculados a partir de multitud de predicciones, una por especie. Por otra parte, resulta difícil e incluso imposible modelizar la distribución de las especies raras, que son las que generalmente determinan gran parte del recambio faunístico entre unidades territoriales próximas. De este modo, para obtener un buen conocimiento a través de la regionalización de las propiedades sinecológicas de las áreas, como la composición faunística, originadas por los patrones de distribución de muchas especies diferentes, proponemos inferir la estructura de estas taxocenosis a partir de aquellas unidades del territorio con inventarios fiables para, posteriormente, obtener modelos predictivos capaces de reflejar la variación faunística del grupo estudiado. Tras extrapolar las variables que resumen la variación faunística a las unidades espaciales sin datos fiables, sería posible realizar una clasificación efectiva de todo el territorio.

En este trabajo proponemos un método capaz de identificar las diferentes regiones faunísticas de una familia de coleópteros, los Escarabeidos (Scarabaeinae) de la Península Ibérica y Baleares, a una resolución de cuadrículas UTM de 50 x $50 \mathrm{~km}$. En primer lugar, identificaremos los diferentes ejes que resumen la variación en la composición de estas especies utilizando para ello la información faunística de aquellas cuadrículas con inventarios bien establecidos. Posteriormente, modelizaremos la distribución de estos ejes mediante distintas variables ambientales y espaciales explicativas (ver, por ejemplo, Lobo \& Martín-Piera, 2002; Lobo \& Hortal, 2003), para después extrapolar los resultados de esta función predictiva al resto del territorio. Finalmente, realizaremos una regionalización faunística de la Península Ibérica utilizando los valores estimados de estos ejes, ponderando la distancia faunística entre cada par de unidades del territorio por la distancia geográfica existente entre ellas, al 


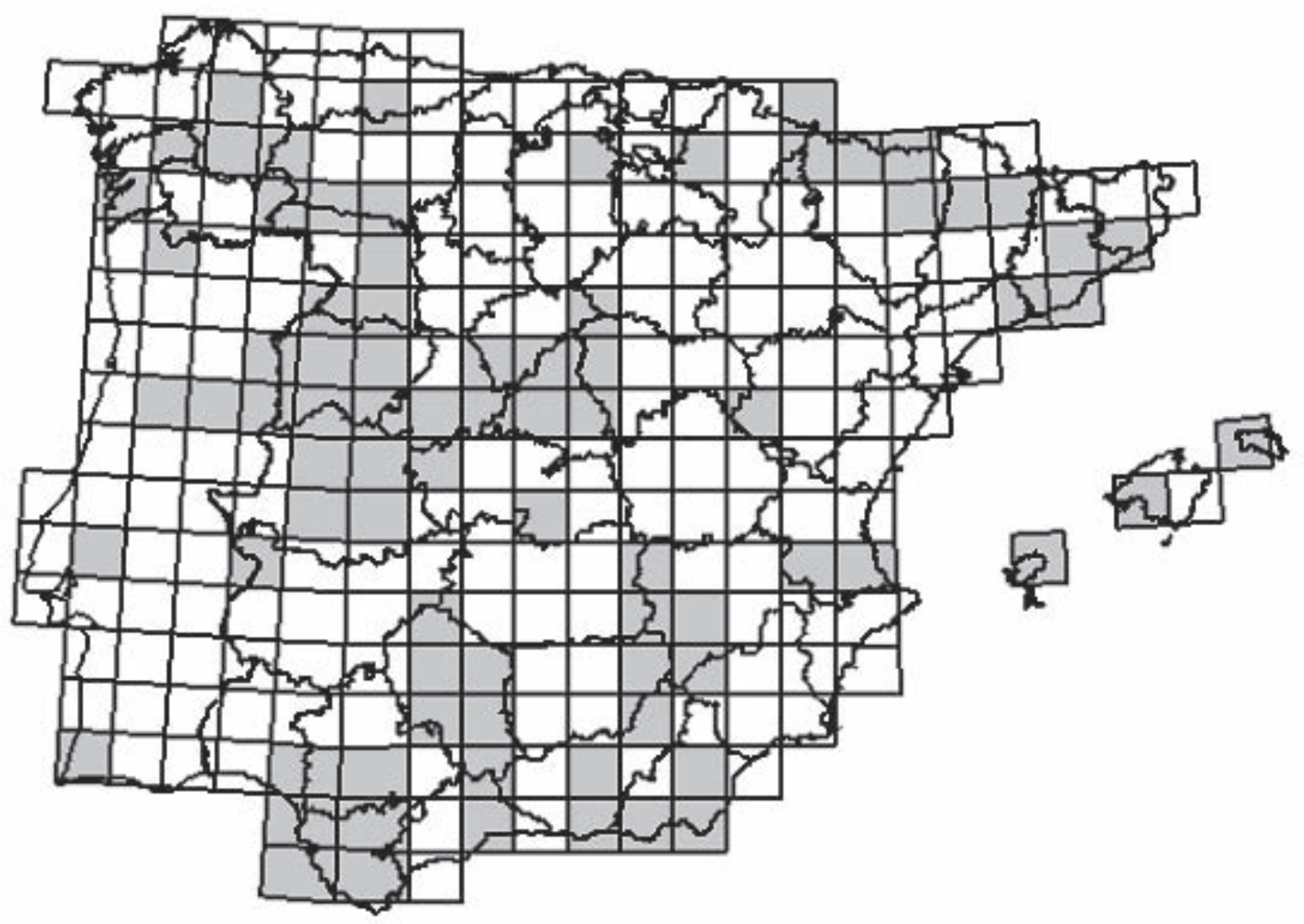

Fig. 1. - 252 cuadrículas UTM de 50x50 km utilizadas como unidades territoriales. En gris se señalan las 82 identificadas como bien muestreadas por Lobo \& Martín-Piera (2002).

Fig. 1. - 252 UTM 50x50 km grid squares used as territorial units. The 82 cells identified as well-sampled by Lobo \& MartínPiera (2002) are shaded.

objeto de agrupar aquellas unidades similares faunísticamente pero, a la vez, espacialmente próximas (Legendre \& Legendre, 1998). El producto final de este proceso es un mapa de las regiones corológicas para los Escarabeidos Ibéricos en el cual se identificarán las especies características de cada región y las variaciones en la riqueza de especies existentes entre ellas.

\section{Métodos}

\section{ORIGEN DE LOS DATOS BIOLÓGICOS}

Como unidades territoriales utilizamos 252 cuadrículas Ibero-Baleares de la malla UTM de 50×50 $\mathrm{km}$ con más de un $15 \%$ de superficie terrestre (Figura 1). Para cada una de estas unidades se obtuvieron los datos de presencia/ausencia de las 53 especies de Escarabeidos que pueden encontrarse en la Península Ibérica (Martín-Piera, 2000). La información corológica proviene de BANDASCA (ver estructura en Lobo \& Martín-Piera, 1991), una base de datos que compila la inmensa mayoría de la información sobre la distribución ibérica de estas especies (ver listado en la Tabla 3). Estudiando en cada cuadrícula la relación asintótica entre el incremento en el número de especies con la acumulación de los registros informáticos (Lobo \& Martín-Piera, 2002), fueron identificadas 82 cuadrículas UTM de 50 x $50 \mathrm{~km}$ con inventarios relativamente bien establecidos (Figura 1).

ESTIMACIÓN DE LOS EJES DE VARIACIÓN FAUNÍSTICA

La matriz rectangular de presencia/ausencia de cada especie en cada cuadrícula (53 especies x 82 cuadrículas) se transformó en una matriz triangular 
de distancias entre cuadrículas, utilizando en este proceso cuatro índices de similitud con diferentes propiedades (ver Legendre \& Legendre, 1998): i) índice de similitud de Jaccard, ii) índice de similitud de Phi (Rohlf, 1998), iii) Distancia Euclídea Binaria (Binary Euclidean Distance; Clustan, 2002) y iv) índice de similitud de Gower (Gower, 1971). Los dos primeros índices difieren en que sus valores tienen en cuenta (Phi) o no (Jaccard) la información proveniente de las dobles ausencias (especies ausentes en las dos cuadrículas comparadas, pero presentes en el territorio analizado). La utilidad de los índices que incorporan las dobles ausencias ha sido puesta en entredicho, con la argumentación de que generalmente no aportan información biogeográfica útil (Buser \& Baroni-Urbani, 1982). Sin embargo, cuando se comparan unidades territoriales con una información faunística bien establecida y en un territorio no muy extenso, como es el caso de la Península Ibérica, la información procedente de las dobles ausencias podría mejorar la capacidad de discriminación entre los distintos conjuntos faunísticos. Los dos últimos índices utilizados poseen la singularidad de permitir otorgar pesos a cada variable para su cálculo, en este caso a cada especie. De este modo, considerando que cuanto mayor es la rareza de una especie mayor es su potencial a la hora discriminar la composición faunística entre las unidades territoriales, hemos utilizado estos índices con el fin de otorgar un peso a cada especie $(1 / n)$ inversamente proporcional al número de presencias $(n)$ en las 82 cuadrículas consideradas como bien muestreadas. La principal diferencia entre estos dos índices es que el espacio obtenido mediante su aplicación es no-euclidiano en el caso del índice de Gower y euclidiano o métrico (que puede representarse fácilmente en un espacio tridimensional) en el caso de la Distancia Euclídea Binaria.

Cada una de las matrices triangulares de proximidad fue sometida a un análisis de ordenación, al objeto de encontrar los principales ejes capaces de describir la variación en la composición faunística. Para este proceso hemos utilizado una técnica de ordenación de comprobada eficacia (Pielou, 1984) que no requiere datos normalmente distribuidos ni matrices de distancia euclidianas, por lo que es capaz de representar eficientemente las distancias observadas en un número pequeño de ejes de variación a partir de cualquier matriz de proximidad (NMDS, Nonmetric Multidimensional Scaling; ver Legendre \& Legendre, 1998). Para determinar el número de dimensiones (ejes de variación) que es conveniente extraer, se analizó la disminución de los valores de stress (una medida de la variabilidad no explicada por los ejes: a menor valor de stress mayor es la capacidad de los ejes para representar la matriz de proximidades) según aumenta el número de dimensiones (scree test; ver StatSoft, 2001).

Tras realizar los análisis de ordenación con cada una de las matrices de proximidad, se seleccionaron los ejes provenientes del índice que mejor fue representado por el NMDS. Para ello se examinó la tasa de disminución en el valor de stress para cada ordenación, seleccionando la configuración que más rápidamente disminuye los valores de stress en el scree test. El número de ejes seleccionado se determinó estudiando los valores de esta gráfica, identificando el momento en que la tendencia de descenso del valor de stress se hace más tendida (StatSoft, 2001). En este análisis, la disminución en el stress al añadir cada nueva dimensión es equivalente a la variabilidad explicada por ese eje.

\section{MODELIZACIÓN DE LOS EJES FAUNíSTICOS}

Los valores de estos ejes de composición faunística, obtenidos para las 82 cuadrículas con inventarios bien establecidos, se extrapolaron al resto del territorio mediante la construcción de un modelo predictivo a partir de los datos de diversas variables ambientales y espaciales en esas mismas cuadrículas (Tabla 1). Para ajustar estas variables en una función predictiva diferente para cada eje faunístico, utilizamos modelos lineares generalizados (GLM; General Linear Models; McCullagh \& Nelder, 1989; Crawley, 1993; Dobson, 1999), usando una función identidad ( $e f=$ intercepto $+a \mathrm{x}$ variable $1+\ldots+h$ x variable $n$ ) para relacionar la variable dependiente (cada uno de los ejes faunísticos) con los valores estandarizados de las variables ambientales y espaciales. Todas las variables se estandarizaron previamente a media 0 y varianza 1 , excepto latitud y longitud, que fueron estandarizadas a la media (ver Legendre \& Legendre, 1998). Posteriormente, las variables ambientales, junto con los nueve términos del polinomio de tercer grado de la latitud y la longitud $\left(b_{1}\right.$ Lat $+b_{2}$ Long + $b_{3}$ Lat $^{2}+b_{4}$ [Lat x Long] $+b_{5}$ Long $^{2}+b_{6}$ Lat $^{3}+b_{7}\left[\right.$ Lat $^{2}$ $\mathrm{x}_{\text {Long }]}+b_{8}\left[\right.$ Lat $\mathrm{x}$ Long $\left.^{2}\right]+b_{9}$ Long $^{3} ;$ Trend Surface Analysis; ver Legendre, 1993), se incluyeron en los análisis por pasos hacia atrás (backward stepwise), en los que las variables con un efecto no significativo $(p<0,05)$ se eliminaron de cada uno de los modelos (StatSoft, 2001).

Las funciones así obtenidas se utilizaron para extrapolar los valores de cada eje a todo el territorio. Para una discusión pormenorizada del método seguido y ejemplos de su aplicación práctica, ver 
Tabla 1.-Códigos utilizados y descripción de las 20 variables ambientales y espaciales utilizadas para desarrollar los mapas predictivos de los ejes faunísticos. Ver origen y descripción en Lobo \& Martín-Piera (2002).

Table 1.- Codes and description of the 20 environmental and spatial variables used to develop the predictive maps for the faunistic axes. See origin and description in Lobo \& MartínPiera (2002).

\begin{tabular}{ll}
\hline Código & Descripción \\
\hline Fisiografia & \\
Altmin & Altitud mínima \\
Altmax & Altitud máxima \\
Difalt & Rango de altitudes \\
Altmed & Altitud media \\
Clima & \\
Tmin & Temperatura Media de las Mínimas mensuales \\
Tmax & Temperatura Media de las Máximas mensuales \\
Tmedann & Temperatura media anual \\
Ampterm & Amplitud térmica \\
Pmedann & Precipitación media anual \\
Pest & Precipitación estival (Jun-Ago) \\
Ampprec & Amplitud anual en las precipitaciones mensuales \\
Hsolann & Porcentaje medio anual de horas de sol \\
Litología & \\
Scal & \\
Sarc & Superficie con suelos básicos \\
Ssil & Superficie con suelos arcillosos \\
Svol & Superficie con suelos silíceos \\
Heterogeneidad Ambiental & Superficie con suelos volcánicos \\
Duso & \\
Dsuel & Diversidad de usos del suelo \\
Coordenadas Geográficas & Diversidad litológica \\
Long & Longitud del punto central \\
Lat & Latitud del punto central \\
\hline & \\
\hline & \\
\hline
\end{tabular}

Hortal \& Lobo (2002), Lobo \& Martín-Piera (2002); Hortal et al. (2001) o Lobo et al. (2002).

\section{CLASIFICACIÓN}

Utilizando los valores extrapolados de cada uno de los ejes faunísticos obtenidos en el paso previo, se construyó una nueva matriz que refleja la variación en la composición entre las 252 cuadrículas, utilizando para ello la distancia euclídea al cuadrado como medida de proximidad (Clustan, 2002). Sin embargo, como cada uno de los ejes faunísticos obtenidos en el análisis de ordenación poseía una capacidad explicativa diferente, esta matriz se construyó asignando a cada eje un peso equivalente a la variabilidad que era capaz de explicar (disminución en el stress).

La composición faunística varía a lo largo del espacio, a medida que unas especies reemplazan a otras, incluso dentro de cada tipo de región faunística. Si asumimos que el efecto de estos cambios en la composición aumenta con la distancia entre las unidades territoriales, es más probable que dos unidades similares faunísticamente, pero separadas espacialmente, presenten determinadas singularidades en su composición. Ello puede deberse a: i) que la conectividad y la persistencia de las poblaciones se vea interrumpida (Araújo \& Williams, 2000; Ferrier, 2002), y/o ii) que la actuación de factores contingentes histórico-geográficos haya provocado pequeñas variaciones entre sus inventarios (Ricklefs \& Schluter, 1993). Debido a ello, se ha calculando el producto de la matriz de distancias geográficas entre las unidades territoriales (distancia euclídea a partir de su latitud y longitud) y la matriz original de distancias faunísticas. Con esta estrategia se pretende obtener una nueva matriz en la que las diferencias faunísticas estén ponderadas espacialmente, de modo que las regiones obtenidas sean aquellas similares en su composición pero, a la vez, contiguas espacialmente.

Los valores de esta nueva matriz de distancias se utilizaron para clasificar el territorio mediante un análisis de agrupamiento (Legendre \& Legendre, 1998), en el que la estrategia de agrupamiento fue el Incremento en la Suma de Cuadrados (método de Ward). Este procedimiento busca minimizar la diferencia entre la suma de las distancias euclídeas al cuadrado de los casos y los valores medios de los grupos a los que estos son asignados. Para estimar qué agrupaciones eran significativas, se comparó el dendrograma o árbol de clasificación obtenido con una serie de 500 árboles generados al azar a partir de la misma matriz de datos. De este modo, los valores de fusión dentro de la jerarquía del árbol de clasificación se compararon con los valores medios obtenidos en las aleatorizaciones, eligiéndose, de entre las significativas, aquella configuración que tenía la mayor diferencia entre ambos valores de fusión (Clustan, 2002). Los resultados de este análisis fueron cartografiados mediante un Sistema de Información Geográfica (Clark Labs, 2001).

\section{Resultados}

\section{EJES DE VARIACIÓN FAUNÍSTICA}

Utilizando cada una de las matrices creadas a partir de los cuatro índices de proximidad, se realizó un NMDS seleccionando un número progresivamente menor de dimensiones. Como los valores de stress de la Distancia Binaria Euclídea disminuyeron más rápidamente al aumentar el número de 


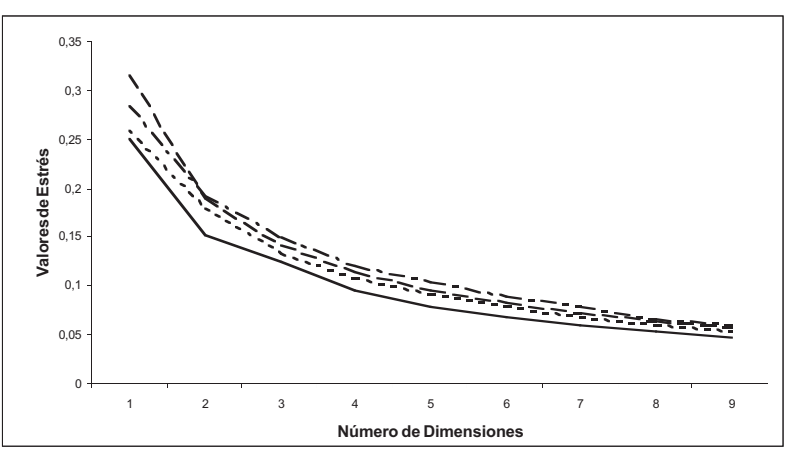

Fig. 2.- Disminución de los valores de stress al aumentar el número de dimensiones en el NMDS de las matrices creadas a partir de los datos de las 82 cuadrículas bien muestreadas por los cuatro índices (ver texto): Jaccard (línea de puntos), Phi (puntos y líneas), Gower (línea discontinua) y Distancia Binaria Euclídea (línea continua).

Fig. 2.- Decrease in the stress values (Y axis) as the number of dimensions extracted with the NMDS increases for the matrices created from data of the 82 well-sampled squares using the four indices (see text): Jaccard (dots), Phi (dots and lines), Gower (discontinuous line) and Binary Euclidean Distance (continuous line).

dimensiones y fueron menores que los de los otros tres índices (Figura 2), se escogió la ordenación producida con este índice. Con cuatro dimensiones (ejes faunísticos, ef), los valores de stress disminuyeron por debajo de 0.1 (stress $=0.094)$ y la tendencia en la disminución de este valor por cada nueva dimensión añadida comenzó a aproximarse a la horizontal (Figura 2). Por ello, utilizamos los cuatro primeros ejes faunísticos (variabilidad explicada: ef $74.9 \%$; ef $9.9 \%$; ef $2.8 \%$; ef $2.9 \%$ ) para describir la variabilidad en los inventarios de Escarabeidos de la Península Ibérica. Los valores de los cuatro ejes estaban normalmente distribuidos.

MODELIZACIÓN PREDICTIVA DE LOS EJES FAUNÍSTICOS

La variabilidad explicada por los cuatro modelos (uno por cada eje) varió desde el $23.5 \%$ del tercer eje, hasta el $55.8 \%$ del primero (Tabla 2). Utilizamos estas funciones para calcular los valores de cada eje en las 252 cuadrículas (cartografiados en la Figura 3). Las predicciones para los cuatro ejes estaban normalmente distribuidas, no existiendo ninguna estructura espacial aparente en los residuos. El primer eje distingue un gradiente de reemplazo faunístico desde el noroeste (valores negativos, principalmente en las zonas montañosas) hacia el sur, donde alcanza valores extremos en las zonas costeras (valores positi- vos). El segundo distingue otro eje de variación montaña-costa, pero en este caso desde todos los sistemas montañosos (valores negativos) hacia las costas atlántica y cantábrica (positivos). El tercer eje presenta valores negativos, principalmente, en el valle del Ebro y en el interior de las dos mesetas, y positivos en el noroeste y algunas zonas de las Sierras Béticas. Finalmente, los valores positivos del cuarto eje aumentan hacia la cornisa cantábrica, y los negativos disminuyen hacia las zonas elevadas adyacentes a las costas del Mediterráneo.

\section{REGIONALIZACIÓN}

Tras obtener la matriz ponderada de distancia faunística pesando la contribución de cada uno de los ejes, obtuvimos la matriz de general de distancias como el producto entre ella y la matriz de distancias espaciales. La clasificación del territorio obtenida con esta matriz agrupó en 8 regiones las 252 cuadrículas ibéricas consideradas (Figura 4) que, a grandes rasgos podrían denominarse como sigue:

1. Región Astur-Galaica, formada por Galicia y Asturias.

2. Región Cántabro-Euskalduna, incluyendo Cantabria, el País Vasco, y parte de Navarra, La Rioja y la provincia de Burgos.

3. Región Ibérico-Castellana, que incluye la submeseta norte, los Sistemas Central e Ibérico, los Montes de León y de Toledo, y los páramos de Guadalajara y Cuenca.

4. Región Luso-Atlántica, formada por la mitad norte de Portugal y un pequeño enclave en el noroeste de Cáceres.

5. Región Pirenaica Oriental, formada por las áreas de alta montaña de los Pirineos centrales y orientales y la Cadena Costera Catalana.

6. Región Levantino-Balear, formada por la desembocadura del Ebro, Levante y el archipiélago Balear.

7. Región Sudoriental, constituida por La Mancha, Albacete, Murcia, Andalucía Oriental, y la Sierra de São Mamede en Portugal.

8. Región Sudoccidental, formada por la mitad sur de Portugal y Andalucía Occidental.

La relación entre las diferentes regiones muestra una clara división latitudinal en dos grandes áreas o dominios: uno que ocupa la mitad sur y el occidente peninsular (regiones 1, 2, 3, 4 y 5), y otro septentrional que se extiende hacia el sur 
Tabla 2.- Resultados de la modelización mediante GLM por pasos hacia atrás para los cuatro ejes faunísticos. Los códigos de las variables en los modelos son los de la Tabla1; g.l. es el número de grados de libertad del modelo; Desv es la desvianza, una medida de la variabilidad de los datos; la significación del cambio en la desvianza ( $\Delta$ Desv) del modelo se identificó mediante un test $F$; Vexp es la proporción de variabilidad explicada por el modelo (ver Hortal \& Lobo, 2002).

Table 2. Results from the GLM backward stepwise modelling procedure for the 4 faunistic axes. Variable codes as in Table 1; g.l. is the number of degrees of freedom of the model; Desv is the deviance, a measure of data variability; significance in the change of deviance of the model ( $\Delta D e s v$ ) was assessed by means of an $F$ test; Vexp is the proportion of total variability explained by the model (see Hortal \& Lobo, 2002).

\begin{tabular}{|c|c|c|c|c|c|c|}
\hline Eje & Modelo & g.l. & Desv & $\Delta D e s v$ & $\boldsymbol{F}$ & Vexp \\
\hline & Nulo & 81 & 81.00 & & & \\
\hline$e f_{1}$ & 0.1926 Dsuel -0.4304 Altmed +0.4985 Hsolann & 78 & 35.79 & 45.21 & 98.51 & 0.558 \\
\hline$e f_{2}$ & $\begin{array}{l}-3.0709 \text { Tmedann }+1.3250 \text { Ampterm }-0.4489 \text { Sarc }-0.3427 \text { Ssil }+ \\
\text { 4.0714Tmin }-0.5176 \text { Altmax }-0.2121 \text { Hsolann }\end{array}$ & 74 & 43.58 & 37.42 & 63.53 & 0.462 \\
\hline$e f_{3}$ & $\begin{array}{l}\text { 0.4484Tmedann }-0.2706 \mathrm{Scal}-0.3109 \text { Ampterm }+0.6587 \text { Altmed }- \\
0.6639 \text { Hsolann }-0.2886 \mathrm{Dsuel}+0.0001 \text { Lat }\end{array}$ & 74 & 61.98 & 19.02 & 22.70 & 0.235 \\
\hline$e f_{4}$ & $\begin{array}{l}\text {-0.3196Ampterm - 0.4683Scal - 0.3163Sarc - 0.2466Altmed - } \\
\text { 0.2905Hsolann - 0.3901Ampprec }\end{array}$ & 75 & 54.47 & 26.53 & 36.54 & 0.328 \\
\hline
\end{tabular}

hasta las estribaciones meridionales de los Sistemas Central e Ibérico y, por el este, hasta las zonas con mayor influencia mediterránea del Valle del Ebro (regiones 6, 7 y 8; ver Figura 4). La similitud faunística entre las regiones del área septentrional sigue también un patrón latitudinal, en donde la región pirenaica se muestra como la más singular, mientras las regiones interiores ( 3 y 4) y atlánticas ( 1 y 2$)$ aparecen claramente asociadas. En el caso del área meridional, las regiones aparecen segregadas longitudinalmente asociándose aquellas regiones con influencia mediterránea (regiones 6 y 7 ).

\section{DIFERENCIAS EN DIVERSIDAD ENTRE REGIONES FAU- NÍSTICAS}

Un gran porcentaje de las especies de Scarabaeinae ibéricas (33 especies, $62 \%$ del total) está presente en seis o mas de las regiones establecidas, de modo que las diferencias faunísticas entre ellas son principalmente debidas a la participación diferencial de unos pocos taxones. Por ejemplo, los dos principales dominios faunísticos comparten el $63 \%$ del total de especies. Sólo 4 especies aparecen exclusivamente en el dominio meridional (S. cicatricosus, O. melitaeus, Ch. irroratus y Ch. furcifer), y 6 son exclusivas del septentrional (O. albarracinus, $O$. stylocerus, $O$. joannae, $O$ coenobita, $O$. nuchicornis y $O$. semicornis). Dentro del dominio septentrional, una única región (la Ibérico-Castellana, $\mathrm{n}^{\circ} 3$ ) alberga el $98 \%$ de las especies presentes en toda su área, poseyendo el resto de las regiones inventarios empobrecidos con resoecto a los de ésta (Tabla 3). Dentro del dominio meridional, la región Levantino-Balear $\left(\mathrm{n}^{\mathrm{o}} 6\right)$ representa un subconjunto faunístico empobrecido del que alberga la región Sudoriental $\left(\mathrm{n}^{\mathrm{0}} 7\right)$, mientras que las diferencias de composición entre las regiones Sudoriental y Sudoccidental se deben únicamente a seis especies (ver Tabla 3).

Las distintas regiones difieren significativamente en la riqueza media de especies por cuadrícula que poseen $\left(F_{(7.74)}=23.36, p=0.001\right)$, aunque los únicos pares de diferencias significativas (Tukey HSD test) corresponden a los de las regiones 3-2, 31, 3-6 y 6-8 (Figura 5), siendo las regiones IbéricoCastellana y Sudoccidental las más ricas en especies dentro de los dominios septentrional y meridional, respectivamente.

\section{Discusión}

Este trabajo demuestra que es posible construir regionalizaciones para cualquier grupo taxonómico, evitando los sesgos en la desigual cobertura geográfica que suele manifestar la información biológica (Dennis \& Thomas, 2000). Para ello es necesario identificar aquellas localidades con inventarios bien establecidos, identificar los principales ejes de composición y elaborar modelos, que como en el caso de otras variables continuas (Guisan \& Zimmermann, 2000), sean capaces de 

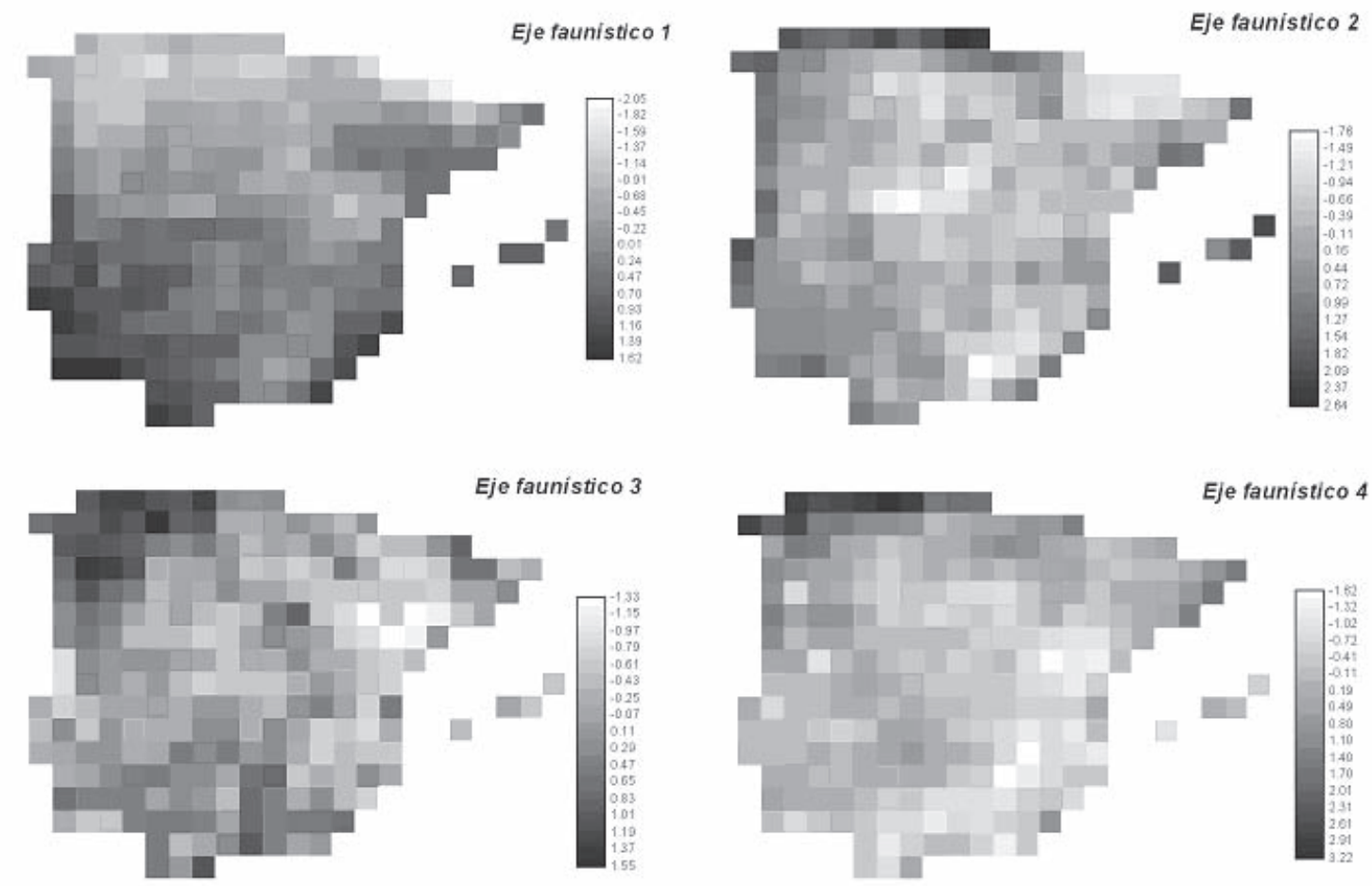

Fig. 3.- Distribución espacial de los 4 ejes faunísticos encontrados mediante NMDS en todas las unidades territoriales, según los valores predichos por los modelos generados mediante GLM (ver Tabla 2).

Fig. 3.- Spatial distribution of the 4 faunistic axes obtained with NMDS for all territorial units following the scores predicted by the GLM models (see Table 2).

predecir su variabilidad espacial. En nuestro caso concreto, aunque la variabilidad explicada por los modelos no sea elevada, el sentido biológico de las regiones producidas nos sugiere que se ha recogido la variabilidad faunística principal de la información de base utilizada. Evidentemente, sería conveniente validar las agrupaciones producidas y para ello sugerimos evaluar la capacidad de esta metodología utilizando matrices con inventarios bien establecidos y seleccionando subconjuntos aleatorios de datos de distinta amplitud. Consideramos especialmente relevante maximizar la proximidad espacial de las localidades a combinar (Legendre \& Legendre, 1998), al objeto de obtener regiones espacialmente continuas y discriminar aquellas otras que, aunque no difieran ostensiblemente en su composición, se encuentren separadas geográficamente. Ello permite, probablemente, obtener regionalizaciones de mayor utilidad en conservación (Ferrier, 2002), ya que las distintas regiones seleccionadas podrían tener una mayor posibilidad de albergar poblaciones genéticamente diferenciadas. Desde un punto de vista estrictamente biogeográfico, este tipo de regionalizaciones podría ser una herramienta útil a la hora de reconocer aquellas áreas con similar composición, pero geográficamente disjuntas, que estuvieron conectadas en un pasado.

El modelo de regionalización obtenido para el caso de los Escarabeidos pone en evidencia la escasa tasa de reemplazo faunístico existente entre distintas localidades Ibero-Baleares (Lobo et al., 1997; Hortal-Muñoz et al., 2000), producida probablemente por la gran capacidad de dispersión de estas especies y/o su gran valencia ecológica (Lobo \& Martín-Piera, 1999). Una sola de las regiones establecidas contiene, aproximadamente, el 75\% del total de especies Ibéricas y en una sola de las cuadrículas UTM de 50 x $50 \mathrm{~km}$ habita, en promedio, el $38 \%$ de las especies Ibéricas. De este modo, las diferencias faunísticas encontradas se deben, prioritariamente, a la contribución desigual de unas pocas especies. En concreto, pueden distinguirse dos grandes dominios faunísticos con similar diver- 


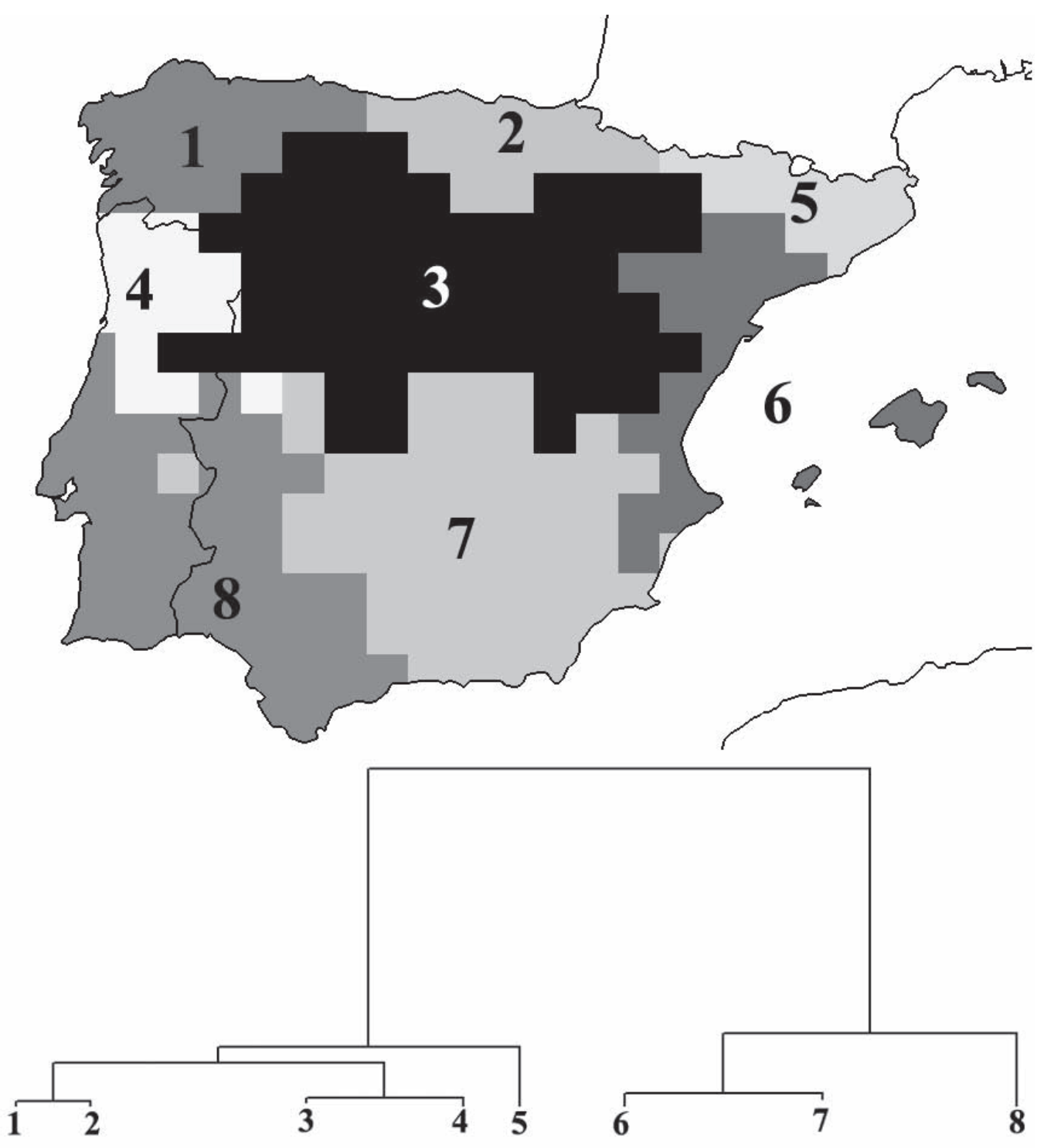

Figura 4.-Regiones faunísticas de los escarabeidos en la Península Ibérica definidas por el análisis de clasificación final (ver texto). Las relaciones entre ellas se muestran en el cladograma inferior.

Figure 4.-Faunistic regions for the scarabaeine in the Iberian Peninsula defined by the final classification analysis (see text). The relationships among them are showed in the tree.

sidad, pero separados latitudinalmente a partir de las estribaciones más mediterráneas de los sistemas Ibérico y Central. En la mitad noroccidental peninsular, las zonas montañosas de los Sistemas Central e Ibérico constituyen uno de los dos territorios de máxima riqueza de especies en la Península Ibérica (Lobo \& Martín-Piera, 2002) en donde, además, aparecen algunos endemismos y se refugian deter- minadas especies de carácter mediterráneo que tienen aquí su límite de distribución septentrional (Martín-Piera, 2001). Así, la región que hemos denominado Ibérico-Castellana, en la cual se encuentra este área de máxima diversidad, posee una riqueza de especies elevada y un número comparativamente superior de especies exclusivas, poseyendo el resto de las regiones faunísticas aso- 
Tabla 3.- Presencia/ausencia y diversidad de las 53 especies de escarabeidos (Col. Scarabaeinae) ibéricas utilizadas en el análisis (ver texto y Lobo \& Martín-Piera, 2002) en las 8 regiones identificadas. Datos extraídos de BANDASCA (ver texto). Los códigos numéricos de las regiones corresponden a los de la Figura 4. Las regiones (columnas) se han ordenado de izquierda a derecha según el número de especies presentes en ellas, y las especies (filas) de arriba a abajo por la cantidad de regiones en las que están presentes. Hemos denominado especies raras a aquellas localizadas tan sólo en 1 ó 2 regiones. La riqueza media por cuadrícula en cada región se calculó a partir tan sólo de los datos de las cuadrículas bien muestreadas presentes en la región.

Table 3.- Presence/absence and diversity of the 53 Iberian scarabaeine species (Col. Scarabaeinae) used in the analysis (see text and Lobo \& Martín-Piera, 2002) in the 8 identified regions. Data extracted from BANDASCA (see text). Numeric codes for the regions as in Figure 4. Regions (columns) have been sorted from left to right following the number of species present in each one of them, and species (rows) from up to down by the number of regions where they are present. We have counted as rare species (Especies raras) those localized only in 1 or 2 regions. Mean richness per square was calculated in each region using only data from the well-sampled squares present in the region.

\begin{tabular}{|c|c|c|c|c|c|c|c|c|c|}
\hline \multirow[b]{2}{*}{ Especies } & \multicolumn{8}{|c|}{ REGIONES } & \multirow[t]{2}{*}{$\mathrm{N}^{0}$ regiones } \\
\hline & 3 & 7 & 8 & 5 & 4 & 6 & 1 & $\overline{2}$ & \\
\hline Bubas bison (L., 1767) & 1 & 1 & 1 & 1 & 1 & 1 & 1 & 1 & 8 \\
\hline Bubas bubalus Olivier, 1811 & 1 & 1 & 1 & 1 & 1 & 1 & 1 & 1 & 8 \\
\hline Caccobius schreberi (L., 1767) & 1 & 1 & 1 & 1 & 1 & 1 & 1 & 1 & 8 \\
\hline Copris lunaris (L., 1758) & 1 & 1 & 1 & 1 & 1 & 1 & 1 & 1 & 8 \\
\hline Euoniticellus fulvus (Goeze, 1777) & 1 & 1 & 1 & 1 & 1 & 1 & 1 & 1 & 8 \\
\hline Euonthophagus amyntas (Olivier, 1789) & 1 & 1 & 1 & 1 & 1 & 1 & 1 & 1 & 8 \\
\hline Gymnopleurus flagellatus (Fabricius, 1787) & 1 & 1 & 1 & 1 & 1 & 1 & 1 & 1 & 8 \\
\hline Onthophagus fracticornis (Preissler, 1790) & 1 & 1 & 1 & 1 & 1 & 1 & 1 & 1 & 8 \\
\hline Onthophagus furcatus (Fabricius, 1781) & 1 & 1 & 1 & 1 & 1 & 1 & 1 & 1 & 8 \\
\hline Onthophagus grossepunctatus (Reitter, 1905) & 1 & 1 & 1 & 1 & 1 & 1 & 1 & 1 & 8 \\
\hline Onthophagus lemur (Fabricius, 1781) & 1 & 1 & 1 & 1 & 1 & 1 & 1 & 1 & 8 \\
\hline Onthophagus opacicollis Reitter, 1893 & 1 & 1 & 1 & 1 & 1 & 1 & 1 & 1 & 8 \\
\hline Onthophagus punctatus (Illiger, 1803) & 1 & 1 & 1 & 1 & 1 & 1 & 1 & 1 & 8 \\
\hline Onthophagus ruficapillus Brulle, 1832 & 1 & 1 & 1 & 1 & 1 & 1 & 1 & 1 & 8 \\
\hline Onthophagus similis (Scriba, 1790) & 1 & 1 & 1 & 1 & 1 & 1 & 1 & 1 & 8 \\
\hline Onthophagus taurus (Schreber, 1759) & 1 & 1 & 1 & 1 & 1 & 1 & 1 & 1 & 8 \\
\hline Onthophagus vacca (L., 1767) & 1 & 1 & 1 & 1 & 1 & 1 & 1 & 1 & 8 \\
\hline Scarabaeus sacer L., 1758 & 1 & 1 & 1 & 1 & 1 & 1 & 1 & 1 & 8 \\
\hline Scarabeus laticollis L., 1767 & 1 & 1 & 1 & 1 & 1 & 1 & 1 & 1 & 8 \\
\hline Sisyphus schaefferi (L., 1758) & 1 & 1 & 1 & 1 & 1 & 1 & 1 & 1 & 8 \\
\hline Copris hispanus (L.,1764) & 1 & 1 & 1 & 1 & 1 & 1 & 1 & 0 & 7 \\
\hline Gymnopleurus mopsus (Pallas, 1781) & 1 & 1 & 1 & 1 & 1 & 1 & 0 & 1 & 7 \\
\hline Gymnopleurus sturmi Mac Leay, 1821 & 1 & 1 & 1 & 1 & 1 & 1 & 0 & 1 & 7 \\
\hline Onthophagus illyricus (Scopolli, 1763) & 1 & 1 & 0 & 1 & 1 & 1 & 1 & 1 & 7 \\
\hline Onthophagus maki (Illiger, 1803) & 1 & 1 & 1 & 1 & 1 & 1 & 1 & 0 & 7 \\
\hline Onthophagus ovatus (L., 1767) & 1 & 0 & 1 & 1 & 1 & 1 & 1 & 1 & 7 \\
\hline Onthophagus verticicornis (Laicharting, 1781) & 1 & 1 & 0 & 1 & 1 & 1 & 1 & 1 & 7 \\
\hline Cheironitis hungaricus (Herbst, 1789) & 1 & 1 & 1 & 1 & 1 & 1 & 0 & 0 & 6 \\
\hline Euoniticellus pallipes (Fabricius, 1781) & 1 & 1 & 1 & 1 & 1 & 1 & 0 & 0 & 6 \\
\hline Euonthophagus gibbosus (L., 1790) & 1 & 1 & 1 & 1 & 0 & 0 & 1 & 1 & 6 \\
\hline Onitis belial Fabricius, 1798 & 1 & 1 & 1 & 1 & 1 & 0 & 1 & 0 & 6 \\
\hline Onitis ion (Olivier, 1789) & 1 & 1 & 1 & 0 & 1 & 1 & 1 & 0 & 6 \\
\hline Scarabaeus puncticollis (Latreille, 1819) & 1 & 1 & 1 & 1 & 0 & 1 & 0 & 1 & 6 \\
\hline Onthophagus coenobita (Herbst, 1783) & 1 & 0 & 0 & 1 & 1 & 0 & 1 & 1 & 5 \\
\hline Onthophagus emarginatus Mulsant, 1842 & 1 & 1 & 1 & 0 & 0 & 1 & 0 & 1 & 5 \\
\hline Onthophagus joannae Goljan, 1953 & 1 & 0 & 0 & 1 & 1 & 0 & 1 & 1 & 5 \\
\hline Onthophagus stylocerus (Graëlls, 1851) & 1 & 0 & 0 & 1 & 1 & 0 & 1 & 1 & 5 \\
\hline Onthophagus hirtus (Illiger, 1803) & 1 & 1 & 1 & 0 & 1 & 0 & 0 & 0 & 4 \\
\hline Onthophagus marginalis Gebler, 1817 & 1 & 1 & 1 & 0 & 1 & 0 & 0 & 0 & 4 \\
\hline Onthophagus merdarius Chevrolat & 1 & 1 & 1 & 0 & 0 & 1 & 0 & 0 & 4 \\
\hline Onthophagus nuchicornis (L., 1758) & 1 & 0 & 0 & 1 & 0 & 0 & 1 & 1 & 4 \\
\hline Scarabaeus semipunctatus Fabricius, 1792 & 0 & 1 & 1 & 1 & 0 & 1 & 0 & 0 & 4 \\
\hline Scarabaeus typhon Fischer-Waldheim, 1823 & 1 & 1 & 0 & 0 & 1 & 0 & 0 & 1 & 4 \\
\hline Onthophagus latigena Dorbigny, 1897 & 1 & 1 & 1 & 0 & 0 & 0 & 0 & 0 & 3 \\
\hline Onthophagus nigellus (Illiger, 1803) & 1 & 1 & 1 & 0 & 0 & 0 & 0 & 0 & 3 \\
\hline Cheironitis irroratus (Rossi, 1790) & 0 & 1 & 1 & 0 & 0 & 0 & 0 & 0 & 2 \\
\hline Euoniticellus pallens (Olivier, 1789) & 1 & 0 & 1 & 0 & 0 & 0 & 0 & 0 & 2 \\
\hline Onthophagus melitaeus (Fabricius, 1798) & 0 & 1 & 1 & 0 & 0 & 0 & 0 & 0 & 2 \\
\hline Onthophagus semicornis (Panzer, 1798) & 1 & 0 & 0 & 0 & 0 & 0 & 1 & 0 & 2 \\
\hline Scarabaeus cicatricosus (Lucas, 1849) & 0 & 1 & 1 & 0 & 0 & 0 & 0 & 0 & 2 \\
\hline Scarabaeus pius (Illiger, 1893) & 1 & 1 & 0 & 0 & 0 & 0 & 0 & 0 & 2 \\
\hline Cheironitis furcifer (Rossi, 1792) & 0 & 0 & 0 & 0 & 0 & 1 & 0 & 0 & 1 \\
\hline Onthophagus albarracinus Baraud, 1979 & 1 & 0 & 0 & 0 & 0 & 0 & 0 & 0 & 1 \\
\hline Número de especies & 48 & 44 & 42 & 37 & 37 & 35 & 33 & 33 & \\
\hline Número de cuadrículas UTM de 50 km & 64 & 52 & 47 & 14 & 15 & 22 & 23 & 15 & \\
\hline Especies raras & 4 & 4 & 4 & $\mathbf{0}$ & $\mathbf{0}$ & 1 & 1 & $\mathbf{0}$ & \\
\hline $\begin{array}{l}\text { Riqueza media por cuadrícula } \\
\pm \text { error estándar }\end{array}$ & $\begin{array}{r}24.0 \\
\pm 1.1\end{array}$ & $\begin{array}{r}20.1 \\
\pm 1.2\end{array}$ & $\begin{array}{l}22.5 \\
\pm 1.6\end{array}$ & $\begin{array}{r}23.2 \\
\pm 2.7\end{array}$ & $\begin{array}{r}21.0 \\
\pm 2.7\end{array}$ & $\begin{array}{l}13.0 \\
\pm 2.4\end{array}$ & $\begin{array}{r}17.3 \\
\pm 2.2\end{array}$ & $\begin{array}{c}15.3 \\
\pm 1.8\end{array}$ & \\
\hline
\end{tabular}




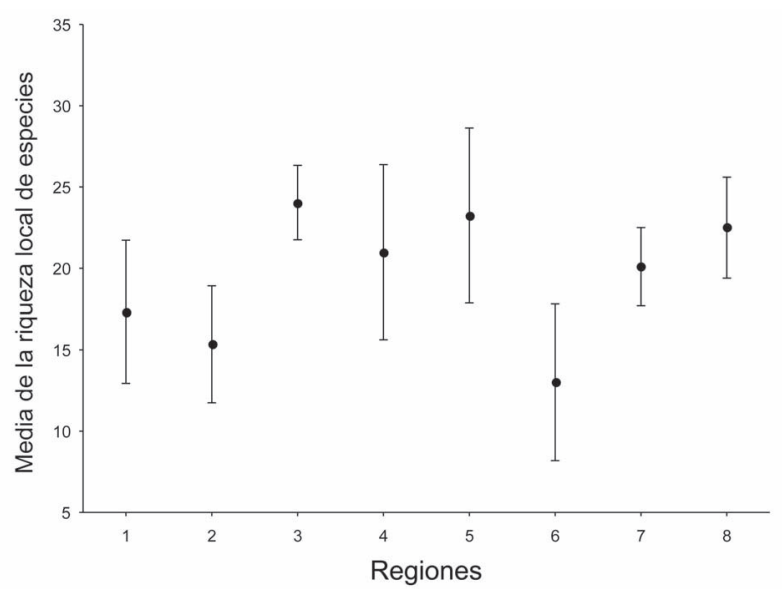

Fig. 5.- Riqueza local media por región. Las líneas representan el intervalo de confianza al 95\%. Los códigos de las regiones se corresponden con los de la Figura 4.

Fig. 5.- Mean local richness per region. Lines represent the 95\% confidence interval. Region codes as in Figure 4.

ciadas inventarios empobrecidos respecto a esta. En las Serranías Béticas y Penibéticas se encuentra el otro territorio de máxima diversidad peninsular (Lobo \& Martín-Piera, 2002), que se encuentra asociado con las regiones faunísticamente diferenciadas de la mitad sudoriental. Como es lógico, la singularidad de estas regiones se debe a la presencia de determinados elementos de afinidad mediterránea y norteafricana. Destaca la pobreza de las regiones Levantina y Balear, la primera propiciada tal vez por los cambios antrópicos recientes, pero la segunda probablemente debida al aislamiento del archipiélago durante los periodos de colonización Ibérica de esta fauna (Martín-Piera \& Lobo, 1992).

\section{AGRADECIMIENTOS}

Este trabajo forma parte de la línea de investigación que manteníamos con nuestro compañero y amigo Fermín y aunque él no ha podido tener el placer de ayudar a escribirlo, sus ideas impregnan todo el manuscrito. Sin él no habría sido posible la recopilación del conocimiento sobre los escarabeidos ibéricos necesario para llevar a cabo un análisis de este tipo, ni el desarrollo del enfoque faunístico-biogeográfico al análisis de la biodiversidad, que es la característica principal de nuestro grupo de trabajo. Por eso, cada vez que escribimos algo, le echamos en falta que nos lea la cartilla. El artículo ha sido financiado por los proyectos REN2001-1136/GLO del MCyT y 07M/0080/2002 de la Comunidad de Madrid. Joaquín Hortal ha podido realizar esta investigación gracias a una beca predoctoral Museo Nacional de Ciencias Naturales/C.S.I.C./Comunidad de Madrid.

\section{Referencias}

AraúJo, M. B. \& Williams, P. H., 2000. Selecting areas for species persistence from occurrence data. Biological Conservation, 96: 331-345.

Araújo, M. B., Humphries, C. J., Densham, P. J., Lampinen, R., Hagemeijer, W. J. M., MitchellJONES, A. J. \& GASC, J. P., 2001. Would environmental diversity be a good surrogate for species diversity? Ecography, 24:103-110.

Austin, M. P., 1999. The potential contribution of vegetation ecology to biodiversity research. Ecography, 22: 465-484.

Austin, M. P. \& Margules, C. R., 1986. Assessing representativeness. In: M. B. Usher (ed.). Wildlife Conservation Evaluation. Chapman \& Hall. London: 45-67.

Bailey, R. G., 1996. Ecosystem Geography. SpringerVerlag. New York. 216 pp.

Bailey, R. G., Pfister, R. D. \& Henderson, J. A., 1978. Nature of land and resource classification: A review. Journal of Forestry, 76:650-655.

Balmford, A. \& Gaston, K. J., 1999. Why biodiversity surveys pay. Nature, 398: 204-205

Bunce, R. G. H., Barr, C. J. \& Whittaker, H. A., 1983. A stratification system for ecological sampling. In: R. M. Fuller (ed.). Ecological Mapping from Ground, Air and Space. Institute of Terrestrial Ecology. Cambridge: 34-46.

Bunce, R. G. H., Barr, C. J., Clarke, R. T., Howard, D. C. \& Lane, A. M. J., 1996. Land classification for strategic ecological survey. Journal of Environmental Management, 47: 37-60.

Buser, M. W. \& BARoni-Urbani, C., 1982. A direct nondimensional clustering method for binary data. Biometrics, 38:351-360.

Clark LABS, 2001. Idrisi 32 Release 2. GIS software package. Clark Labs, Worcester, MA.

Clustan, Ltd., 2002. Clustan Graphics Version 5.25. Clustan Ltd.

Crawley, M. J., 1993. GLIM for Ecologists. Blackwell Scientific Publications.

Dennis, R. L. H. \& HaRDY, P. B., 1999. Targeting squares for survey: predicting species richness and incidence of species for a butterfly atlas. Global Ecology and Biogeography, 8: 443-454.

Dennis, R. L. H. \& Thomas, C. D., 2000. Bias in butterfly distribution maps: the influence of hot spots and recorder's home range. Journal of Insect Conservation, 4: 73-77.

De PABlo, C. L. \& PinedA, F. D., 1985. Análisis multivariante del territorio para su cartografía ecológica. Ensayo preliminar en la provincia de Madrid. Anales de Geografia de la Universidad Complutense, 5: 235-260. 
De Pablo, C. L., Gómez Sal, A. \& Pineda, F. D., 1987. Élaboration automatique d'une cartographie écologique et son évaluation avec des paramètres de la théorie de l'information. L'Espace Géographique, 2: 115128.

de Pablo, C. L., Martín de Agar, P., Gómez Sal, A. \& PINEDA, F. D., 1988. Descriptive capacity and indicative value of territorial variables in ecological cartography. Landscape Ecology, 1: 203-211.

DoADrio, I., 1988. Delimitation of areas in the Iberian Peninsula on the basis of freshwater fishes. Bonner Zoologische Beitrage, 39: 113-128.

Dobson, A., 1999. An introduction to Generalized Linear Models. Chapman \& Hall/CRC. New York. $174 \mathrm{pp}$.

Elena Rosselló, R., Ferreiro, G. T., Andrade, J. L. A. \& Palomares, O. S., 1990. Clasificación biogeoclimática territorial de España: Definición de EcoRegiones. Ecología. Fuera de Serie, 1: 59-79.

Elena Rosselló, R., Ferreiro, G. T., Castejón Ayuso, M. A. \& Palomares, O. S., 1993. Clasificación Biogeoclimática Territorial de España (CLATERES): Una herramienta de utilidad para planificar la reforestación de España. Montes, 33: 50-56.

Elena Rosselló, R., CAstejón Ayuso, M. A., SÁnchez Serrano, F. \& Ferreiro, G. T., 1997. Clasificación Biogeoclimática de España Peninsular y Balear. Atlas CLATERES. INIA, Ministerio de Agricultura, Pesca y Alimentación, Madrid. 446 pp.

Faith, D. P., Walker, P. A., Margules, C. R., Stein, J. \& NATERA, G., 2001. Practical application of biodiversity surrogates and percentage targets for conservation in Papua New Guinea. Pacific Conservation Biology, 6: 289-303.

FERRIER, S., 2002. Mapping Spatial Pattern in Biodiversity for Regional Conservation Planning: Where to from Here? Systematic Biology, 51: 331-363.

Ferrier, S., Drielsma, M., Manion, G. \& Watson, G., 2002. Extended statistical approaches to modelling spatial pattern in biodiversity in north-east New South Wales. II. Community-level modelling. Biodiversity and Conservation, 11: 2309-2338.

FISHER, B. L., 1999. Improving inventory efficiency: a case study of leaf litter ant diversity in Madagascar. Ecological Applications, 9: 714-731.

García-Barros, E., Gurrea, P., Luciáñez, M. J., Martín Cano, J., Munguira, M. L., Moreno, J. C., SAINZ, H., SANZ, M. J. \& SimÓN, J. C., 2002. Parsimony analysis of endemicity and its application to animal and plant geographical distributions in the Ibero-Balearic region (western Mediterranean). Journal of Biogeography, 29: 109-124.

Gavilán, R. G., Fernández-GonzÁlez, F. \& Blasi, C., 1998. Climatic classification and ordination of the Spanish Sistema Central: relationships with potential vegetation. Plant Ecology, 139: 1-11.
Guisan, A. \& Zimmermann, N. E., 2000. Predictive habitat distribution models in ecology. Ecological Modelling, 135: 147-186.

Gower, J. C., 1971. A general coefficient of similarity and some of its properties. Biometrics, 27: 857-871.

Haila, Y. \& Margules, C. R., 1996. Survey research in conservation biology. Ecography, 19: 323-331.

Hengeveld, R., 1990. Dynamic Biogeography. Cambridge University Press. Cambridge. 263 pp.

Hermida, J., Outeiro, A. \& Rodríguez, T., 1994. Biogeography of terrestrial gastropods in north-west Spain. Journal of Biogeography, 21: 207-217.

Hortal, J. \& LoBo, J. M., 2002. Una metodología para predecir la distribución espacial de la diversidad biológica. Ecología (n.s.), 16: 151-178 + 14 figuras.

Hortal, J., Lobo, J. M. \& Martín-Piera, F., 2001. Forecasting insect species richness scores in poorly surveyed territories: the case of the Portuguese dung beetles (Col. Scarabaeinae). Biodiversity and Conservation, 10: 1343-1367.

Hortal-Muñoz, J., Martín-Piera, F. \& Lobo, J. M., 2000. Dung beetle geographic diversity variation along a western Iberian latitudinal transect (Col., Scarabaeidae). Annals of the Entomological Society of America, 93: 235-243.

Izco, J., 1984. Madrid Verde. Ministerio de Agricultura, Pesca y Alimentación/Comunidad de Madrid. Madrid. 517 pp.

Lawton, J. H., 1999. Are there general laws in ecology? Oikos, 84: 177-192.

LEGENDRE, P. 1993. Spatial autocorrelation: trouble or new paradigm? Ecology, 74: 1659-1673.

LEGENDRE, P. \& LEGENDRE, L., 1998. Numerical Ecology, Second english edition. Elsevier. Amsterdam. 870 pp.

Lobo, J. M. \& Hortal, J., 2003. Modelos predictivos: Un atajo para describir la distribución de la diversidad biológica. Ecosistemas, 2003/1. Disponible en http://www.aeet.org/ecosistemas/investigacion3.htm.

Lobo, J. M. \& MARTín-PierA, F., 1991. La creación de un banco de datos zoológico sobre los Scarabaeidae (Coleoptera: Scarabaeoidea) íbero-baleares: una experiencia piloto. ELYTRON, 5: 31-38.

Lobo, J. M. \& MARTín-PierA, F., 1999. Between-group differences in the Iberian dung beetle species-area relationship (Coleoptera, Scarabaeidae). Acta Oecologica, 20: 587-597.

Lobo, J. M. \& Martín-Piera, F., 2002. Searching for a predictive model for species richness of Iberian dung beetle based on spatial and environmental variables. Conservation Biology, 16: 158-173.

Lobo, J.M., Sanmartín, I. \& Martín-Piera, F., 1997. Diversity and spatial turnover of dung beetle (Coleoptera, Scarabaeoidea) communities in a protected area of south Europe (Doñana National Park, Huelva, Spain). ELYTRON, 11: 71-88. 
Lobo, J. M., Lumaret, J. P. \& Jay-Robert, P., 2002. Modelling the species richness of French dung beetles (Coleoptera, Scarabaeidae) and delimiting the predicitive capacity of different groups of explanatory variables. Global Ecology and Biogeography, 11: 265-277.

Mac Nally, R., Bennett, A. F., Brown, G. W. Lumsden, L. F., Yen, A., Hinkley, S., Lilywhite, P. \& WARD, D., 2002. How well do ecosystem-based planning units represent different components of biodiversity? Ecological Applications, 12: 900-912.

Márquez, A. L., Real, R. \& Vargas, J. M., 2001. Methods for comparison of biotic regionalizations: the case of pteridophytes in the Iberian Peninsula. Ecography, 24: 659-670.

Martín-PierA, F., 2000. Familia Scarabaeidae. In: F. Martín-Piera \& J. I. López-Colón. Coleoptera, Scarabaeoidea I. Museo Nacional de Ciencias Naturales, Consejo Superior de Investigaciones Científicas. Madrid: 207-432.

MARTín-PIERA, F., 2001. Area networks for conserving Iberian insects: A case study of dung beetles (Col., Scarabaeoidea). Journal of Insect Conservation, 5: 233-252.

Martín-PierA, F. \& LoBo, J. M., 1992. Los Scarabaeoidea Laparosticti (Coleoptera) del archipiélago balear. Nouvelle Revue d'Entomologie (N.S.), 9: 15-28.

McCullagh, P. \& Nelder, J. A., 1989. Generalized Linear Models, 2nd edition. Chapman \& Hall. Cambridge. $511 \mathrm{pp}$.

Moreno Saiz, J.C., Castro Parga, I. \& Sainz Ollero, H., 1998. Numerical analyses of distributions of Iberian and Balearic endemic monocotyledons. Journal of Biogeography, 25: 179-194.

Morrone, J. J., 2001. Biogeografia de América Latina y el Caribe. CYTED/ORCYT-UNESCO/SEA. Zaragoza. $148 \mathrm{pp}$.

Palomo, L. J., Vargas, J. M. \& Jiménez-Gómez, M. P., 1994. Distribution patterns in Iberian Peninsula rodents. Polish Ecological Studies, 20: 497-502.

Peters, D. \& Thackway, R., 1998. A new biogeographic regionalisation for Tasmania. Technical Report NR002, Commonwealth of Australia, Parks \& Wildlife Service. Hobart. 42 pp.

Pielou, E. C., 1984. The Interpretation of Ecological Data. Wiley. New York. 288 pp.

Powell, G. V. N., Barborak, J. \& Rodríguez, S. M., 2000. Assessing representativeness of protected natural areas in Costa Rica for conserving biodiversity: a preliminary gap analysis. Biological Conservation, 93: 35-41.

Puente, A. I., Altonaga, K., Prieto, C. \& Rallo, A., 1998. Delimitation of biogeographical areas in the Iberian Peninsula on the basis of Helicoidea species (Pulmonata: Stylommatophora). Global Ecology and Biogeography Letters, 7: 97-113.
RAPOPORT, E. H., 1975. Areografía. Estrategias Geográficas de las Especies. Fondo de Cultura Económica. México. 214 pp.

Real, R., Guerrero, J. C. \& Ramírez, J. M., 1992. Identificación de las fronteras bióticas significativas para los anfibios en la cuenca hidrográfica del sur de España. Doñana, Acta Vertebrata, 19: 53-70.

Real, R., Márquez, A. L., Guerrero, J. C., Vargas, J. M. \& Palomo, L. J., 1996. Modelos de distribución de los insectívoros en la Península Ibérica. Doñana, Acta Vertebrata, 23: 123-142.

RiCKLEFS, R. E. \& SChluter, D., 1993. Species diversity: Regional and historical influences. In: R.E. Ricklefs \& D. Schluter (eds.). Species Diversity in Ecological Communities. Historical and Geographical Perspectives. The University of Chicago Press. Chicago: 350-363.

Rivas-MartíneZ, S., 1987. Memoria del Mapa de Series de Vegetación de España. Ministerio de Agricultura, Pesca y Alimentación. Madrid. 268 pp. + 30 mapas 1:400.000.

Rodríguez, T., Trigo, D. \& DíAz Cosín, D., 1997. Biogeographical zonation of the western Iberian Peninsula on the basis of the distribution of earthworm species. Journal of Biogeography, 24: 893-901.

RoHLF, J., 1998. NTSYSpc version 2.02i. Exeter Software. Setauket, NY.

Scott, J. M., Davis, F. W., Csuti, B., Butterfield, B., Groves, C., Anderson, H., Caicco, S., D'Erchia, F., Edwards, T. C., Ulliman, J. \& Wright, R. G., 1993. Gap analysis: a geographic approach to protection of biological diversity. Wildlife Monographs, 123: 1-41.

Sainz Ollero, H. \& Hernández Bermejo, J. E., 1985. Sectorización fitogeográfica de la Península Ibérica e islas Baleares: la contribución de su endemoflora como criterio de semejanza. Candollea, 40: 485-508.

StATSOFt, Inc., 2001. STATISTICA (data analysis software system). StatSoft Inc. Tulsa.

Vargas, J. M., Real, R. \& Guerrero, J. C., 1998. Biogeographical regions of the Iberian peninsula based on freshwater fish and amphibian distributions. Ecography, 21: 371-382.

Viejo, J. L., Ibero, C., De Silva, C. \& Martín, J., 1992. Las regiones lepidopterológicas del norte de España. Boletín de la Real Sociedad Española de Historia Natural (Sección Biológica), 88: 223-233.

Wohlgemuth, T., 1996. Biogeographical regionalization of Switzerland based on floristic data: how many species are needed? Biodiversity Letters, 3: 180-191. 